gung fühlen können. Dabei bietet die Hausleitung des Bundesministeriums der Justiz schon ein deutliches Signal, der Kollege Diwell weiß es aus seiner Zeit als Staatssekretär. Die Hausleitung besteht aus drei Personen. Es sind immerhin eine Ministerin und eine beamtete Staatssekretärin. Das ist schon eine gute Quote, die allerdings durch meine Anwesenheit als Parlamentarischer Staatssekretär dort im Hause ein wenig geschmälert wird. Wichtiger ist aber vielleicht die Auswertung unseres Personalverwaltungssystems, die wir gerade aktuell vorgenommen haben. Diese hat ergeben, dass der Frauenanteil in unserem Ministerium insgesamt stolze 58 Prozent beträgt. Im höheren Dienst sind es immerhin 44 Prozent. Und jetzt habe ich überlegt, ob ich den Rest meines Manuskriptes lieber streichen sollte, weil viele Präsidentinnen und Präsidenten der Gerichte anwesend sind, aber ich erwähne es doch: $\mathrm{Zu}$ unserem Geschäftsbereich gehören auch rund 240 Richterinnen und Richter an drei obersten Bundesgerichten, nämlich dem Bundesgerichtshof, dem Bundesverwaltungsgericht und dem Bundesfinanzhof. Hier fällt die Statistik folgendermaßen aus: Am Bundesgerichtshof sind von den 129 Richterstellen 28, also knapp 22 Prozent, mit Frauen besetzt. Bei den Vorsitzenden ist der Frauenanteil nicht einmal halb so hoch. Nur zwei von fünfzehn dieser Stellen sind mit Frauen besetzt. Beim Bundesverwaltungsgericht ist der Frauenanteil mit rund 26 Prozent zwar insgesamt höher, der Anteil bei den Vorsitzenden liegt derzeit allerdings bei null Prozent. Aber wir haben ja die große Freude, dass das Bundesverwaltungsgericht von einer hier heute anwesenden - Präsidentin geleitet wird, worüber ich mich sehr freue. Der Bundesfinanzhof schließlich hat mit 20 Prozent zwar den geringsten Frauenanteil insgesamt, aber ein Viertel der Vorsitzendenstellen ist mit Frauen besetzt. Das ist doch schon einigermaßen beachtlich.

Diese kurze Bilanz fällt also durchwachsen aus. Sie ist nicht ganz schlecht, aber sie ist auch noch nicht gut genug. Daher, Frau Präsidentin Wagner, will ich das Grußwort dazu nutzen, Ihnen zu versichern, Ihre Ankündigung von vorhin, dass Sie mit Ihrem Kongress, uns, dem öffentlichen Dienst als Arbeitgeber, schon die richtigen Instrumente empfehlen werden, nehme ich sehr ernst. Wir kommen gerne darauf zurück. Das gilt für die gesamte Hausleitung, für meine Person, der ich hier Gast sein darf, für die beamtete Staatssekretärin Frau Dr. Grundmann und für die Ministerin Frau LeutheusserSchnarrenberger, von der ich Sie sehr herzlich grüßen darf. Dankeschön.

\title{
Es gibt viel zu tun - auch bei den Frauen in Leitungs- positionen des öffentlichen Dienstes
}

\section{Jann Jakobs \\ Oberbürgermeister, Potsdam}

Sehr geehrte Frau Präsidentin Wagner, Herr Minister Dr. Schöneburg, Herr Staatssekretär Dr. Stadler, meine sehr verehrten Damen und Herren,

als Oberbürgermeister heiße ich Sie ganz herzlich willkommen zu Ihrem 39. Bundeskongress in der Landeshauptstadt Potsdam. Ich finde es richtig klasse, dass der Brandenburger Landesverband des djb diese Einladung ausgesprochen hat. Ich finde es auch toll, dass die Veranstaltung nicht etwa im Neuen Palais, im Cecilienhof oder womöglich in Sanssouci stattfindet, sondern hier in der Schiffbauergasse. Denn dieses Gelände dürfte weniger bekannt sein und ich finde es sehr schön, dass Sie sich hier heute in der Schinkelhalle versammeln. Ich finde es auch deshalb sehr erfreulich, weil Potsdam in der Regel mit wunderbaren Schlössern in Verbindung gebracht wird. Das ist auch gut so und eine gute Werbemarke. Aber es wäre wesentlich zu kurz gegriffen, wenn man Potsdam auf diese drei Bauwerke reduzieren würde. Insoweit haben wir ja auch dafür Sorge getragen, dass hier in der Schiffbauergasse ein Gegenakzent gesetzt wird - architektonisch, aber auch kulturell.

Hier versammelt sich auf der einen Seite die Kreativwirtschaft im VW Design Center. In Steinwurf-Nähe sagt man bes- ser nicht, weil das Center ein Glashaus in unmittelbarer Nähe ist. Auf der anderen Seite befindet sich die Deutschlandzentrale von Oracle. Diese Gewerbebetriebe in Verbindung mit Kultur ist etwas ganz Herausragendes. Wir haben hier unser Theater neu eröffnen können vor ein paar Jahren. Das hat wirklich Strahlkraft und wenn ich das sage, meine Damen und Herren, dann bedeutet das schon etwas in der unmittelbaren Umgebung Berlins. Und die Strahlkraft wirkt auch in Richtung Berlin. Ich bin immer sehr stolz darauf, wenn mir die Zahlen vorgelegt werden und ich sagen kann „Siehste, ein Drittel kommt aus Berlin“. Wir sagen hier, Berlin ist bedeutend, deshalb ist der Papst auch dort, aber Potsdam ist schön, deshalb sind Sie hier, meine Damen.

Potsdam zeichnet sich dadurch aus, dass es eine wachsende Stadt ist, eine der wenigen Städte im Osten Deutschlands, in Deutschland überhaupt, die wächst. Wir haben mittlerweile rund 157.000 Einwohner. Wenn man den Statistikern glauben darf, dann wird dieses Wachstum auch in den nächsten Jahren zu verzeichnen sein. Wir sollen in den nächsten zehn Jahren rund 170.000 Einwohner haben. Deshalb sind wir auch kräftig dabei, einen entsprechenden Wohnungsbau zu realisieren, damit die Einwohnerinnen und Einwohner dann auch alle hier wohnen können.

Der Tourismus ist eine ganz wichtige Einnahmequelle in dieser Stadt. Wir haben rund 900.000 Übernachtungen pro 


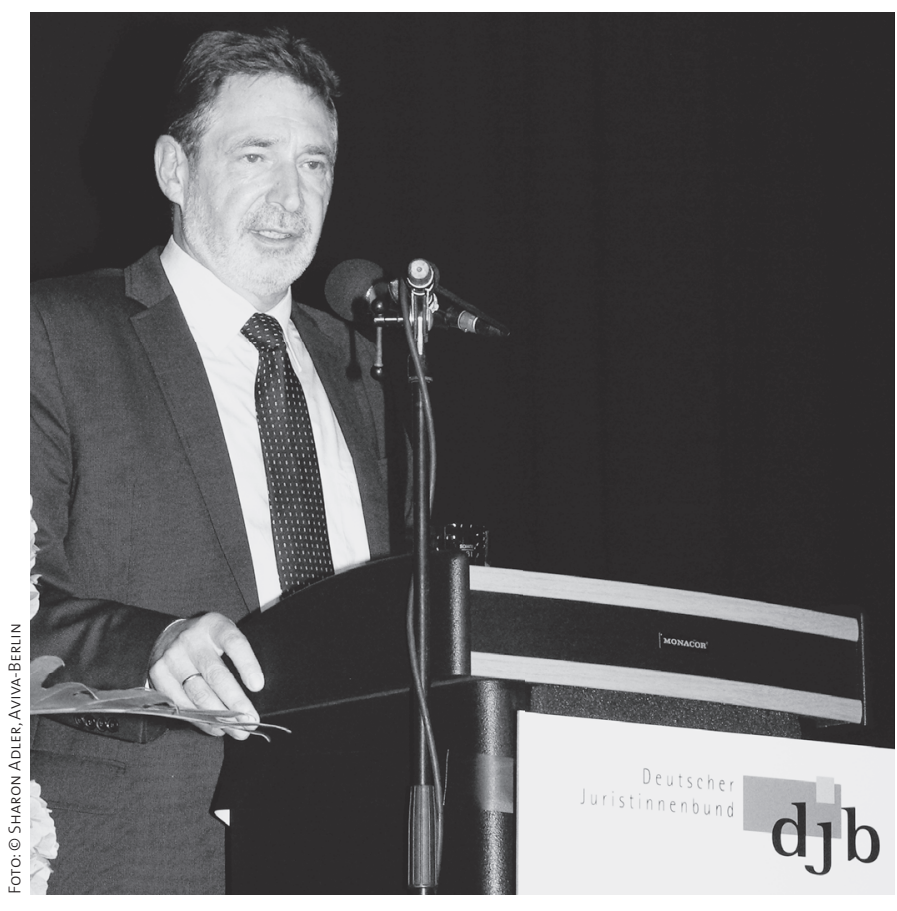

A Oberbürgermeister Jann Jakobs spricht ein Grußwort.

Jahr. Ich habe es selbst kaum glauben wollen, aber jährlich kommen 18 Millionen Tagesbesucher in die Stadt. Die machen das natürlich so, dass sie vorwiegend ein paar Tage in Berlin sind und dann nach Potsdam kommen. Wir arbeiten daran, dieses Verhältnis umzukehren. Sie machen es genau richtig, wie ich finde. Sie tagen in Potsdam, Sie übernachten auch hier, vielleicht fahren Sie am Sonntagnachmittag nach Berlin. Ich finde das vollkommen in Ordnung. Wissen Sie, es ist auch so, dass statistisch zum Ausdruck gebracht wird, dass jede Besucherin und jeder Besucher im Schnitt 30 Euro pro Tag hierlässt und da sind die Unterbringungskosten nicht mit eingerechnet. Ich sage das nur, meine Damen und Herren, damit Sie eine Anregung erhalten hinsichtlich der Ausgaben, die Sie in den nächsten Tagen hier zu tätigen haben. Das ist eine wichtige Einnahmequelle, aber es wäre auch wiederum zu kurz gegriffen, wollte man Potsdam nur auf seine Sehenswürdigkeiten reduzieren. Zugegebenermaßen ist die Stadt kein traditionsreicher Gerichtsstandort, aber sie ist ein wichtiger Gerichtsstandort hier in Brandenburg mit Verfassungsgericht,
Landgericht, Landesarbeitsgericht. Alle genannten Gerichte befinden sich in der Landeshauptstadt Potsdam. Ich lade einmal im Jahr auch die Gerichtspräsidenten zu einem Austausch ein, um darüber zu sprechen und konkret zu verabreden, was hier auf kommunalpolitischer Ebene für die Gerichte auf den Weg gebracht werden soll.

Herr Staatssekretär, ich habe mich natürlich auch gefragt bei dem Thema „Gleichberechtigung“, wie es denn eigentlich in meiner Verwaltung aussieht und ich beginne auch „oben“. Also, der Oberbürgermeister ist ein Mann, das sehen Sie, meine Damen und Herren. Dann kommt die sogenannte Beigeordnetenriege, da sind wir paritätisch besetzt, zwei Frauen und zwei Männer. Das Verhältnis hat sich aber zulasten der Frauen verschlechtert, denn bis vor Kurzem waren es drei Frauen und ein Mann. Dann geht es weiter mit den Leitungsebenen. Mir wurde aufgeschrieben, dass 8,1 Prozent der Männer Leitungspositionen inne haben und nur 7,1 der Frauen. Man könnte nun sagen, naja, da sind wir auf dem Weg. Aber nein, die Situation ist vielmehr so, dass erstens dieser Zustand seit Jahren stagniert und zum zweiten muss man diese Zahl wiederum in Relation zu den Beschäftigten der Stadtverwaltung setzen. Wir haben unter den Beschäftigten zwei Drittel Frauen und ein Drittel Männer. Insoweit haben wir in der Tat noch Nachholbedarf. Es gibt einen Gleichstellungsplan der Landeshauptstadt Potsdam und wir arbeiten emsig daran, dieses Verhältnis zu verbessern. Die meisten Beschäftigen des öffentlichen Dienstes dürfte es in den Kommunalverwaltungen geben. Daher sind sie ein ganz wichtiger Schlüssel, um dafür Sorge zu tragen, dass Frauen in Leitungspositionen kommen. Es gibt hier in den nächsten Jahren sicherlich viel zu tun.

Um über die richtigen Strategien nachzudenken, ist Potsdam der richtige Ort. Dazu wünsche ich Ihnen, meine sehr verehrten Damen, sehr viel Freude, eine anregende Umgebung und viel Biss und Durchsetzungskraft, um Ihre Ziele in den nächsten Jahren durchzusetzen. Potsdam ist ja für das eine oder andere Grundsatzabkommen durchaus bekannt, nicht immer in positiver Hinsicht, aber es könnte ja auch ein Potsdamer Abkommen zur Gleichberechtigung in den öffentlichen Verwaltungen geben. Damit würden wir gerne in die Geschichte eingehen. In diesem Sinne wünsche ich Ihnen viel Freude, viel Spaß und kommen Sie gerne wieder her. Vielen Dank!

\section{Menschenwürde, Freiheit, Gleichheit}

\section{Festvortrag für den 39. Jahreskongress des Deutschen Juristinnenbundes $2011^{1}$}

\section{Prof. Dr. Susanne Baer, LL.M. \\ Richterin des Bundesverfassungsgerichts, Karlsruhe}

Mit dem Deutschen Juristinnenbund ist das so eine Sache. Er kommt als ganz harmlose berufsständische Vereinigung daher, nimmt dann aber in einer Vielzahl von Rechtsfragen eminent politisch Stellung. Er wirkt so ganz und gar bodenständig, bürgerlich, macht dann aber Aktionen, die sogenannte bürgerliche Kreise durchaus irritieren. Dazu gehören sicherlich diese anstrengenden Fragen auf den Versammlungen der Aktiengesellschaften: Warum sitzt da keine Frau? Wie ist das mit den

\footnotetext{
1 Die Vertragsfassung wurde beibehalten. Ich bedanke mich für die Recherchen bei Marieke Michel.
} 